\title{
Zeitliche Veränderungen und Einflussfaktoren des Rauchverhaltens im Hinblick auf die Umsetzung von Präventionsmaßnahmen
}

\author{
Temporal Changes and Determinants of Smoking Habits with Respect to \\ Prevention
}

\section{Zusammenfassung}

In entwickelten Ländern gilt Rauchen als einer der wichtigsten Risikofaktoren für das Auftreten kardiovaskulärer Erkrankungen und als bedeutendste Einzelursache für ein frühzeitiges Versterben. In der vorliegenden Untersuchung wird das Rauchverhalten unter den Teilnehmern der MONICA-Surveys S1 (1984/85), S2 (1989/90) und S3 (1994/95) analysiert in Bezug auf Prävalenz, Einflussfaktoren, Zeittrends und präventive Konsequenzen. Ein wesentliches Ziel dieser Studie lag in der Identifizierung von Risikogruppen, die im Rahmen von Präventionsmaßnahmen angesprochen werden sollten, um längerfristig eine Reduzierung der hohen Mortalität an Herz-Kreislauf-Erkrankungen zu erreichen. Bei den Teilnehmern des MONICA-Surveys S3 (1994/95) lag der Anteil der Raucher mit 31,2\% bei Männern und 22,0\% bei Frauen niedriger als im Bundesdurchschnitt (Bundesgesundheitssurvey 1998: $37,4 \%$ und 29,9\%). Unabhängig vom Alter oder von soziodemografischen Faktoren wiesen Frauen stets einen geringeren Anteil an Rauchern auf als Männer. In beiden Geschlechtern wurde eine starke Abhängigkeit der Raucherprävalenz vom Ausbildungsgrad, vom Familienstand und von der beruflichen Stellung ermittelt. Im Zeittrend wiesen Männer eine positive Entwicklung mit sinkender Raucherprävalenz auf, während bei Frauen ein ansteigender Anteil an Raucherinnen beobachtet worden ist. Die Motivation zum Ändern des Rauchverhaltens wurde bei Män-

\section{Abstract}

Smoking habits are seen as one of the most important risk factors in developed countries for the incidence of cardiovascular diseases and as the major single cause of premature death. The present study analysed smoking habits in participants of the MONICA-Surveys S1 (1984/85), S2 (1989/90) and S3 (1994/95) concerning prevalence, associated factors, time trends and preventive consequences. An essential aim of these studies was to identify risk groups, that should be addressed in prevention campaigns leading to a reduction of the high mortality due to cardiovascular diseases. The participants of the MONICA-Survey S3 (1994/95) had lower proportions of smokers (31.2\% for men and $22.0 \%$ for women) as compared to estimated proportions of the entire German population (National health survey 1998: 37.4\% und $29.9 \%$ ). Women had always a lower proportion of smokers than men in each age group or sociodemographic factor. Strong associations of smoking habits with educational levels, family status and occupational position were observed for men and women. A positive time trend with decreasing smoking prevalence was determined for men. In contrast, an increase in the prevalence of female smokers was observed. The intention to change smoking habits was associated with different factors in men and women. For men, mainly „external“ factors like social status or physical activity were important. Women seemed to be more

Anmerkung

Die MONICA/KORA-Studiengruppe besteht aus: KORA: H.-E. Wichmann (Sprecher), H. Löwel, C. Meisinger, T. Illig, R. Holle, J. John und deren Mitarbeitern, die verantwortlich für das Design und die Durchführung der KORA-Studien sind; MONICA: U. Keil (Studienleiter), A. Döring, B. Filipiak, H. W. Hense, H. Löwel, J. Stieber und den Mitarbeitern, die verantwortlich für das Design und die Durchführung der MONICA-Studien waren.

Institutsangaben

${ }^{1}$ GSF - Forschungszentrum für Umwelt und Gesundheit, Institut für Epidemiologie, Neuherberg

${ }^{2}$ Institut und Poliklinik für Psychosomatische Medizin, Med. Psychologie und Psychotherapie des Klinikums rechts der Isar der Technischen Universität München

${ }^{3}$ Augsburg Central Hospital, MONICA/KORA Myocardial Infarction Registry, Augsburg

Korrespondenzadresse

Jens Baumert, Dipl.-Math. · GSF - Forschungszentrum für Umwelt und Gesundheit, Institut für Epidemiologie . Postfach 1129 · 85758 Neuherberg · E-mail: baumert@gsf.de

Bibliografie

Gesundheitswesen 2005; 67 Sonderheft 1: S46 -S50 @ Georg Thieme Verlag KG Stuttgart • New York DOI $10.1055 / \mathrm{s}-2005-858242$

ISSN 0949-7013 
nern und Frauen von unterschiedlichen Faktoren beeinflusst. Während bei Männern vor allem „externe“ Faktoren wie der Sozialstatus oder die körperliche Aktivität eine Rolle spielten, ließen sich Frauen eher bei „internen“ Faktoren wie körperlichen Beschwerden oder einer negativen Selbsteinschätzung der eigenen Gesundheit zur Änderung des Rauchverhaltens bewegen. Sinnvoll wären differenzierte geschlechts-, alters- und bildungsspezifische Maßnahmen und Empfehlungen, um unterschiedliche Personengruppen gezielter zu erreichen.

\section{Schluisselwörter}

Rauchverhalten · Prävention · Zeittrends · Raucherentwöhnung influenced by „internal“ matters (somatic complaints, negative self-rated health). Sex-, age- and educational status-specific activities and recommendations are considered to be essential for targeting different person groups in a more focused way.

\section{Key words}

Smoking habits $\cdot$ prevention $\cdot$ time trends $\cdot$ smoking cessation

\section{Einleitung}

Rauchen wird als einer der wichtigsten Risikofaktoren für das Auftreten von mehr als 40 Krankheiten und als bedeutendste Einzelursache für ein frühzeitiges Versterben in entwickelten Ländern angesehen [1, 2]. Darüber hinaus wird Rauchen zunehmend auch in Entwicklungsländern als ernsthafte Gefahr für die Gesundheit der Bevölkerung betrachtet [3]. Die WHO geht davon aus, dass gegenwärtig weltweit jährlich etwa fünf Millionen Menschen an den Folgen ihres Rauchverhaltens sterben, was in etwa jedem zehnten Todesfall eines Erwachsenen entspricht [4]. In Deutschland wird derzeit von etwa 120000 Todesfällen pro Jahr aufgrund von Tabakkonsum ausgegangen [5]. In verschiedenen Studien wurde Rauchen als einer der wichtigsten Risikofaktoren für das Auftreten verschiedener kardiovaskulärer Erkrankungen ermittelt [1]. So wird für Raucher das Risiko, einen tödlichen oder nichttödlichen Herzinfarkt zu erleiden, als mehr als doppelt so hoch eingeschätzt wie bei Personen, die nie geraucht haben [6]. Das Risiko für das Auftreten eines Herzinfarkts steigt hierbei mit der Anzahl gerauchter Zigaretten pro Tag an: Raucher, die pro Tag im Mittel 20 oder mehr Zigaretten rauchen, haben ein über dreifach erhöhtes Risiko gegenüber Personen, die nie geraucht haben; während bei Rauchern mit weniger als 20 Zigaretten pro Tag das entsprechende Risiko bei etwa 2 liegt.

Die Bedeutung der kardiovaskulären Risikofaktoren und speziell des Zigarettenrauchens für das Herzinfarktrisikos wird ganz aktuell durch die 52 Länder einschließende INTERHEART-Studie unterstrichen, in welcher insgesamt 15000 Herzinfarktpatienten (Fälle) mit einer gleichgroßen Anzahl an Kontrollen aus der Bevölkerung verglichen wurden [7]. Aufgrund dieser starken Auswirkungen auf die Gesundheit gelten Maßnahmen, Raucher zum Ändern ihres Rauchverhaltens zu motivieren, als ein wichtiger Aspekt zur Prävention von verschiedenen, insbesondere aber von kardiovaskulären Erkrankungen. Die WHO hat deshalb Initiativen gestartet, um die Prävalenz des Tabakkonsums zu reduzieren [4]. Hierzu werden auf öffentlicher Ebene ein Verbot von direkter oder indirekter Tabakwerbung, eine deutliche Erhöhung der Tabaksteuer oder rauchfreie Zonen am Arbeitsplatz und an öffentlichen Einrichtungen empfohlen. Nicht alle Bevölkerungsgruppen können hierbei gleichermaßen durch Präventionsmaßnahmen erreicht werden. Vor allem für Zielgruppen mit erhöhter Prävalenz oder auffälligen, gesundheitlich ungünstigen Verhaltensweisen sind Präventionsmaßnahmen sinnvoll. Deshalb ist eine Ausrichtung auf spezifische Personengruppen zu empfeh- len. Für die Planung und Umsetzung von Präventionsmaßnahmen zum Ändern des Rauchverhaltens mit dem Ziel, längerfristig eine Reduzierung z.B. der hohen Mortalität an Herz-KreislaufErkrankungen zu erreichen, stellen deshalb Kenntnisse über die Abhängigkeit zwischen Rauchverhalten und spezifischen Faktoren eine notwendige Voraussetzung dar.

In der vorliegenden Untersuchung werden Ergebnisse verschiedener Studien zusammengefasst, in denen anhand einer bevölkerungsbezogenen Studienpopulation hinsichtlich des Rauchverhaltens Analysen zur Prävalenz, zu Einflussfaktoren, zu Zeittrends und zu Aspekten der Prävention durchgeführt wurden. Das Hauptaugenmerk lag in der Identifizierung von Risikogruppen, die im Rahmen von Präventionsmaßnahmen angesprochen werden sollten.

\section{Methodik}

\section{Studienpopulation}

Die vorliegende Arbeit basiert auf Daten, die primär im Rahmen des internationalen WHO-MONICA-Projektes (Monitoring of Trends and Determinants in Cardiovascular Diseases) in der Region Augsburg (Stadt Augsburg und Landkreise Augsburg und Aichach-Friedberg) erhoben worden sind [8]. In den Jahren 1984/85 (S1), 1989/90 (S2) und 1994/95 (S3) wurden aus den Einwohnermelderegistern zufällig ausgewählte Personen eingeladen, um die alters- und geschlechtsspezifische Prävalenz der kardiovaskulären Risikofaktoren nach einem standardisierten Untersuchungsprotokoll zu ermitteln. Außerdem wurde allen Probanden Blut abgenommen, und ein Teil dieser Blutproben wurde für spätere Analysen eingelagert. Insgesamt haben 13427 Personen (6725 Männer, 6702 Frauen; Response 77\%) im Alter von 25 - 74 Jahren an mindestens einer der 3 MONICA-Surveys teilgenommen.

\section{Erhobene Daten}

Im Rahmen von standardisierten persönlichen Interviews wurden unter anderem soziodemografische Faktoren (Geschlecht, Alter, Bildungsgrad, Familienstand, Berufstätigkeit), Angaben zum sozialen Netzwerk, zu bekannten kardiovaskulären Risikofaktoren (Adipositas, Alkoholkonsum, körperliche Aktivität, Hypertonie), zu psychodiagnostischen Parametern (Depression, Schlafstörungen, somatische Beschwerden) und zur Selbsteinschätzung der Gesundheit erhoben. 
Zur Beschreibung des Rauchverhaltens wurden die Teilnehmer in 4 Gruppen eingeteilt:

- Regelmäßiger Raucher: Teilnehmer raucht regelmäßig, d.h. gewöhnlich mindestens eine Zigarette pro Tag.

- Gelegentlicher Raucher: Teilnehmer raucht gelegentlich, d.h. gewöhnlich weniger als eine Zigarette pro Tag.

- Ehemaliger Raucher: Teilnehmer hat früher einmal Zigaretten geraucht.

- Nieraucher: Teilnehmer hat noch nie Zigaretten geraucht.

Außerdem wurde nach der mittleren Anzahl der gerauchten $\mathrm{Zi}$ garetten pro Tag, dem Alter bei Beginn des Zigarettenrauchens, der Zigarettensorte und dem Rauchverhalten der Eltern gefragt. Die regelmäßigen Raucher wurden gebeten, Angaben hinsichtlich unternommener oder geplanter Versuche, ihr Rauchverhalten zu verändern oder zu beenden, zu machen. Hierbei wurden die folgenden 4 Punkte erhoben:

1. Raucher äußert Wunsch, gerne mit dem Rauchen aufzuhören

2. Raucher hat versucht, in den letzten 12 Monaten mit dem Rauchen aufzuhören

3. Raucher hat versucht, in den letzten 12 Monaten weniger zu rauchen

4. Raucher hat vor, in den nächsten 12 Monaten das Rauchverhalten zu ändern

Raucher wurden als unmotiviert eingeschätzt, wenn sie alle 4 Punkte negativ beantworteten, und umgekehrt als veränderungsbereit, wenn sie alle 4 Punkte positiv beantworteten.

\section{Ergebnisse und Diskussion}

\section{Prävalenz des Rauchens}

Die Prävalenz des Zigarettenrauchens lag bei den Teilnehmern von S3 bei 31,2\% für Männer und 22,0\% für Frauen [9]. Der Bundesgesundheitssurvey 1998 gab für Deutschland bei Personen mit einem Alter von 25 bis 69 Jahren insgesamt eine höhere Raucherprävalenz von 37,4\% (Männer) und 29,9\% (Frauen) an [10]. Die Abweichungen in der Prävalenz des Rauchens bei den Teilnehmern von S3 im Vergleich zum Bundesgesundheitssurvey lagen wohl hauptsächlich in Unterschieden in der Bevölkerungsstruktur beider betrachteten Populationen begründet. Im internationalen Vergleich nimmt Deutschland hinsichtlich des Anteils von Rauchern in der Bevölkerung einen geringeren bis mittleren Rang ein [11]. In Abb.1 ist für verschiedene Altersgruppen getrennt nach Männern und Frauen die jeweilige Raucherprävalenz für die Teilnehmer von S3 angegeben. Es zeigte sich in beiden Geschlechtern eine stark abnehmende Prävalenz mit zunehmendem Alter.

Der altersspezifische Unterschied in der Prävalenz war in beiden Geschlechtern hochsignifikant $(\mathrm{p}<0,05)$. Im Bundesgesundheitssurvey 1998 und in internationalen epidemiologischen Studien wurde ebenso dieser deutliche Zusammenhang zwischen dem Alter und der Prävalenz des Rauchens ermittelt.

Eine Begründung für die Abnahme der Raucherprävalenz mit zunehmendem Alter liegt zum einen vermutlich an der höheren Mortalität von Rauchern, d.h., Personen, die bereits in jungen

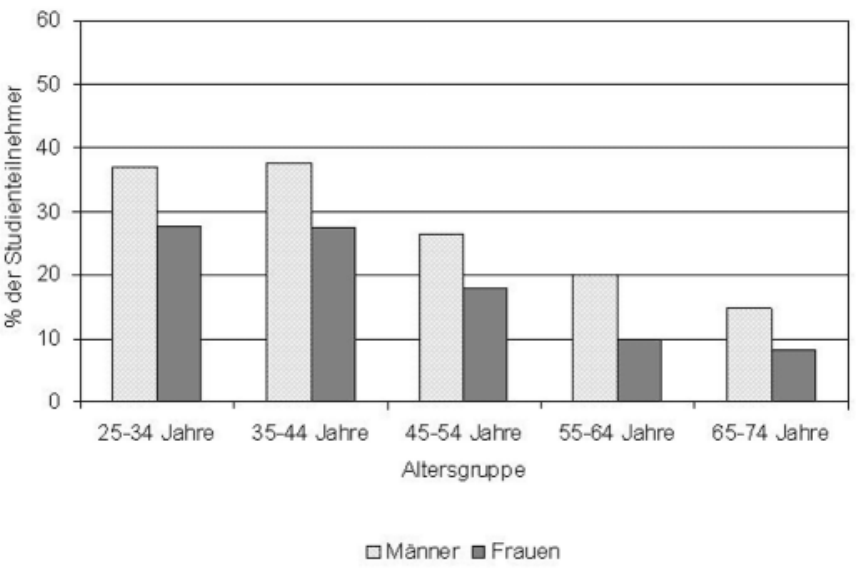

Abb. 1 Prävalenz des Rauchens in Abhängigkeit von der Altersgruppe für Männer und Frauen, MONICA-Survey Augsburg 1994/95 (S3).

Jahren anfangen zu rauchen, erreichen seltener als Nichtraucher höhere Altersgruppen. Vor allem aber sind zum anderen Menschen mit zunehmendem Alter sensibilisierter für gesundheitliche Gefahren. Im Gegensatz zu vielen anderen kardiovaskulären Risikofaktoren dürfte allgemein bekannt sein, dass Rauchen erhebliche Auswirkungen auf die Gesundheit haben kann. Auch dürften gruppendynamische Bedingungen wie beispielsweise Imitation von Vorbildern, die oftmals zum Rauchverhalten beitragen, mit zunehmendem Alter eine geringere Rolle spielen.

Neben dem Alter und Geschlecht hängt der Anteil der Raucher auch stark von soziodemografischen Faktoren ab. Im Rahmen der MONICA-Surveys wurde in verschiedenen Studien analysiert, inwieweit das Rauchverhalten abhängig ist vom Ausbildungsstand, sozialen Umfeld bzw. Netzwerk und beruflichem Status [12 - 14]. Hierbei zeigte sich, dass mit steigender Ausbildung der Anteil der Zigarettenraucher abnahm. Sowohl bei Männern als auch bei Frauen war die Raucherprävalenz am niedrigsten bei jenen Teilnehmern mit dem höchsten Ausbildungsgrad. Die bildungsspezifischen Unterschiede im Rauchverhalten waren hierbei bei Männern ausgeprägter als bei Frauen. Hinsichtlich des beruflichen Status fand sich bei männlichen Beamten und Landwirten der geringste Anteil an Rauchern, während bei Arbeitern und leitenden Angestellten dieser Anteil am höchsten war. Bei den Frauen waren es vor allem einfache und leitende Angestellte, die rauchten. Deutlich weniger als alle anderen Berufsgruppen rauchten bei den Frauen Beamtinnen. Hinsichtlich des sozialen Netzwerks wurde eine starke Abhängigkeit zum Rauchverhalten ermittelt: Je mehr soziale Kontakte jemand aufwies, umso geringer fiel der Anteil der Raucher aus. Der Einfluss des sozialen Netzwerks auf die Raucherprävalenz war sehr ähnlich bei Männern und Frauen.

Abschließend bleibt festzuhalten, dass unabhängig vom Alter oder von soziodemografischen Faktoren Frauen stets einen geringeren Anteil an Zigarettenrauchern aufwiesen als Männer.

\section{Trends}

In einer Studie zu 10-Jahres-Trends von kardiovaskulären Risikofaktoren in der Studienregion der MONICA-Kohorte [9] wurde eine positive Entwicklung mit sinkender Raucherprävalenz bei Männern festgestellt: Während im ersten Survey S1 noch 35,7\% 

Gegensatz dazu stand die Entwicklung bei Frauen mit signifikant leicht zunehmender Tendenz zu rauchen (18,5\% auf 22,0\%). Eine entsprechende Entwicklung wurde auch bei der mittleren Anzahl gerauchter Zigaretten pro Tag ermittelt. Bei Männern war hierbei eine Abnahme zu verzeichnen, wohingegen bei Frauen eine Zunahme beobachtet worden ist. In Abb. $\mathbf{2}$ ist das Rauchverhalten in Abhängigkeit von verschiedenen Altersgruppen nach Untersuchungsjahr für Männer und Frauen dargestellt. Es zeigte sich bei Männern vor allem in den jüngeren und älteren Altersgruppen eine starke Abnahme des Anteils an Rauchern im Vergleich zu S1 $(p<0,05)$, während bei Frauen in den beiden mittleren Altersgruppen (35-54 Jahre) ein Anstieg des Anteils an Raucherinnen vor allem zwischen S1 und S2 zu verzeichnen war $(\mathrm{p}<0,05)$.

In einer weiteren Studie wurden unterschiedliche Zeittrends im Rauchverhalten nach Bildungsgrad ermittelt [15]. Hierbei zeigte sich, dass der Anstieg der Raucherprävalenz bei Frauen vor allem auf Raucherinnen mit niedrigem Bildungsgrad zurückzuführen ist. Im Bundesgesundheitssurvey 1998 und in verschiedenen anderen Ländern sind ähnliche Trends in der Raucherprävalenz ermittelt worden [10,11]. Ein Effekt der bislang durchgeführten Präventionsmaßnahmen zum Rauchen hinsichtlich sinkender Raucherquoten lässt sich somit allenfalls bei Männern feststellen.

\section{Prävention}

In einer Studie zu den Absichten, das Rauchverhalten zu ändern oder ganz mit dem Rauchen aufzuhören, wurden die hierzu gemachten Angaben der regelmäßigen Raucher analysiert [16]. Etwa $60 \%$ der regelmäßigen Raucher gaben an, sie würden gerne mit dem Rauchen aufhören. Ein Anteil von etwa 30\% hat in den letzten 12 Monaten mindestens einmal versucht, mit dem Rauchen aufzuhören. Es traten hierbei nur geringfügige geschlechtsoder altersspezifische Unterschiede auf. Während Frauen signifikant häufiger in den letzten 12 Monaten Versuche unternommen haben, weniger zu rauchen, äußerten Männer öfter den Wunsch, mit dem Rauchen ganz aufzuhören, als Frauen $(p<0,05)$ (Abb. 3).

Bislang ist wenig bekannt, durch welche Faktoren Raucher zu einer Entscheidung zum Ändern des Rauchverhaltens motiviert werden. Deshalb wurden jene Raucher mit keinerlei Absicht, ihr Rauchverhalten zu ändern, mit jenen Rauchern, die motiviert waren, mit dem Rauchen aufzuhören oder zumindest weniger zu rauchen, in Bezug auf ihre soziodemografischen und psychosozialen Eigenschaften miteinander verglichen. Es zeigten sich hierbei keine signifikanten geschlechts- oder altersspezifischen Unterschiede bei der Motivation, das Rauchverhalten zu ändern. Allerdings wurden in einer multivariaten Analyse bei Männern andere Einflussfaktoren als bei Frauen ermittelt. Bei Männern erhöhte sich die Motivation, das Rauchverhalten zu ändern, vor allem bei Vorliegen von konkreten Beeinträchtigungen im psychosozialen Bereich (Schlafstörungen, Depressionen, fehlende körperliche Belastbarkeit) oder Einflüssen aus dem sozialen Umfeld (Familienstand oder Schulbildung). Männer, die mit einer Partnerin zusammenlebten, wollten weitaus häufiger mit dem Rauchen aufhören als allein lebende Männer. Auch war bei Männern ein niedriger oder mittlerer Bildungsgrad im Vergleich zu

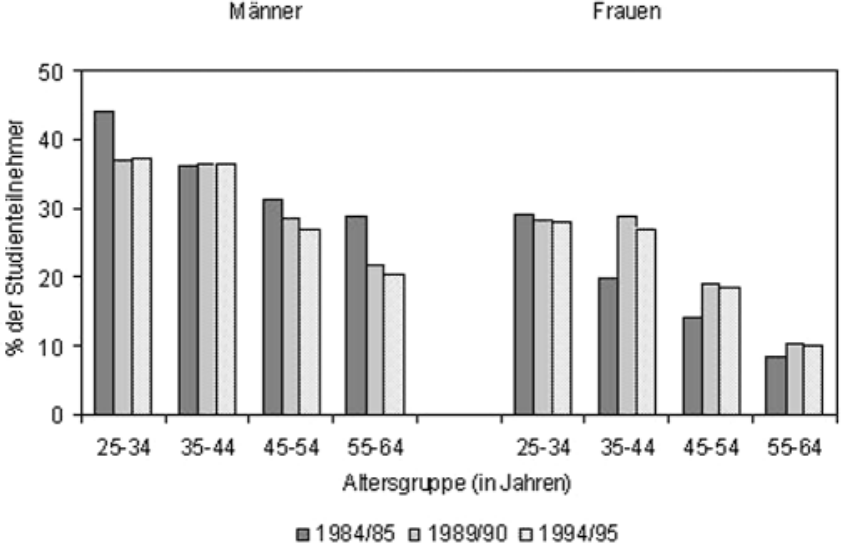

Abb. 2 Rauchverhalten im Zeittrend in Abhängigkeit von der Altersgruppe für Männer und Frauen, MONICA-Surveys Augsburg 1984/85 (S1), 1989/90 (S2) und 1994/95 (S3) (Daten aus Hense et al., 1998 [9]).

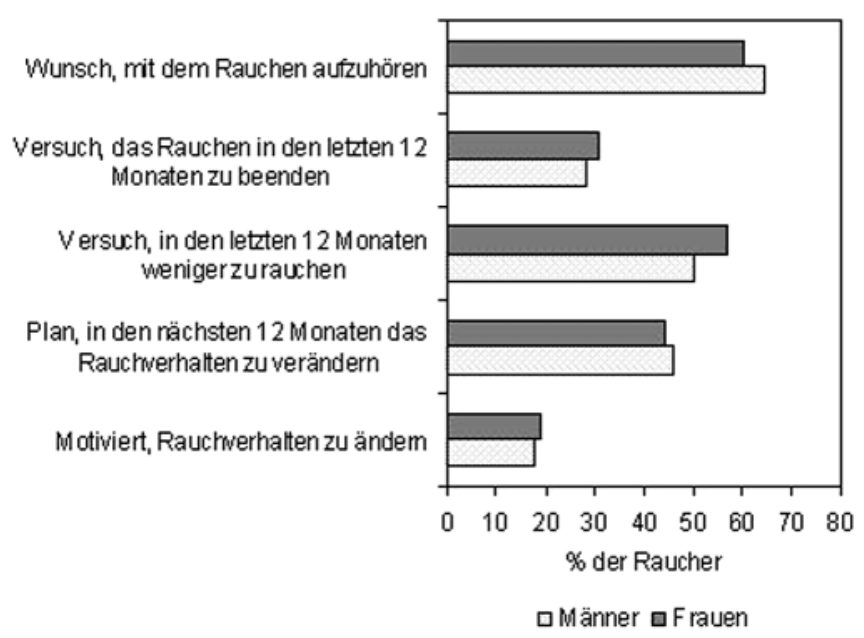

Abb. 3 Änderungen im Rauchverhalten bei regelmäßigen Rauchern für Männer und Frauen, MONICA-Surveys Augsburg 1984/85 (S1), 1989/90 (S2) und 1994/95 (S3) (Daten aus Ladwig et al., im Druck [16]).

einem hohen Bildungsgrad mit einem höheren Anteil an motivierten Rauchern assoziiert. Bei Frauen hingegen lagen die entsprechenden Gründe vor allem in internen körperlichen Signalen (somatische Beschwerden, negative Einschätzung der eigenen Gesundheit). Darüber hinaus verstärkte auch ein späteres Einstiegsalter und eine geringere Anzahl an gerauchten Zigaretten pro Tag bei Frauen die Motivation zu Änderungen des Rauchverhaltens.

Die Motivation zum Ändern des Rauchverhaltens wird bei Männern und Frauen von unterschiedlichen Faktoren beeinflusst. Während bei Männern vor allem „externe“ Faktoren wie der Sozialstatus oder die körperliche Aktivität eine Rolle spielen, lassen sich Frauen eher bei „internen“ Faktoren wie bei körperlichen Beschwerden oder bei einer negative Selbsteinschätzung der eigenen Gesundheit zur Änderung des Rauchverhaltens bewegen. Aufgrund der geschlechtsspezifischen Unterschiede hinsichtlich des Einflusses der psychosozialen Faktoren auf die Motivation, das Rauchverhalten zu ändern, sind unterschiedliche Strategien für Männer und Frauen zu empfehlen. Insgesamt weisen die Ergebnisse dieser Studie auch darauf hin, dass bei Rauchern oft- 
mals erst das Auftreten von gesundheitlichen Problemen zu einem verstärkten Nachdenken über die Rauchgewohnheiten führt.

Ein weiterer wichtiger Aspekt für die Umsetzung von zukünftigen Präventionsprogrammen liegt in der eher geringen Motivation bei Raucherinnen mit einem frühen Einstiegsalter ( $<21$ Jahre) oder einem hohen Zigarettenkonsum ( $\geq 20$ Zigaretten pro Tag). Diese Frauen sind, da sie länger und intensiver rauchen, eher gefährdet, eine kardiovaskuläre Erkrankung zu erleiden, als andere Raucherinnen.

Die Ergebnisse der Studie zur Prävention unterstreichen die Notwendigkeit von fortwährenden Bemühungen um intensive Aufklärung der Bevölkerung hinsichtlich des Zusammenhangs von Rauchen mit der erhöhten Gefahr von verschiedenen Erkrankungen [16].

\section{Ausblick}

Neben Aktivitäten auf öffentlicher Ebene wie beispielsweise den von der WHO empfohlenen Initiativen, die auf eine allgemeine Reduzierung der Raucherprävalenz in der Bevölkerung abzielen, sind Präventionsmaßnahmen zum Rauchen durch gezieltes Ansprechen spezifischer Zielgruppen sinnvoll. Unterschiedliche Zeittrends im Rauchverhalten sind für Männer und Frauen bei den Teilnehmern der MONICA-Surveys ermittelt worden. Detaillierte Analysen von Zeittrends in Abhängigkeit von individuellen Merkmalen, insbesondere von soziodemografischen Faktoren wie Bildungsgrad oder Sozialstatus, könnten mögliche Unterschiede in den Auswirkungen von Interventionsmaßnahmen aufzeigen und damit die Entwicklung und Verbesserung der Inhalte und Schwerpunkte von zukünftigen Präventionsprogrammen mit dem Ziel der Änderung von Rauchgewohnheiten unterstützen. Darüber hinaus sind weitere Analysen zu Assoziationen zwischen dem Rauchverhalten und genaueren soziodemografischen Faktoren, die im Rahmen der umfangreichen Erhebung im KORA-Survey S4 (1999/2001) erfasst worden sind, notwendig, um zielgerichtete Kampagnen entwickeln zu können.

\section{Danksagung}

Die MONICA/KORA-Untersuchungen zum Rauchen wurden gefördert durch die GSF und das BMBF - Bundesministerium für Bildung und Forschung/Helmholtz-Gemeinschaft VH-VI-143 und NGFN.

\section{Literatur}

${ }^{1}$ Doll R. Fifty years of research on tobacco. Journal of Epidemiology and Biostatistics 2000; 5: 321 - 329

${ }^{2}$ Peto R, Lopez AD, Boreham J et al. Mortality from tobacco in developed countries: indirect estimation from national vital statistics. Lancet 1992; 339: 1268 - 1278

${ }^{3}$ Collishow NE, Lopez AD. Prevalence of cigarette smoking in developing countries (editorial). Tob Control 1995; 4: 327

${ }^{4}$ World Health Organisation. Tobacco Smoke and Involuntary Smoking. IARC Monographs 83. Lyon: IARC, 2004

${ }^{5} \mathrm{John}$ U, Hanke M. Tabakrauch-attributable Mortalität in den deutschen Bundesländern. Das Gesundheitswesen 2001; 63: 363 - 369

${ }^{6}$ Keil U, Liese AD, Hense HW et al. Classical risk factors and their impact on incident non-fatal and fatal myocardial infarction and all-cause mortality in southern Germany. Results from the MONICA Augsburg cohort study 1984 - 1992. Eur Heart J 1998; 19: 1197-1207

${ }^{7}$ Yusuf S, Hawken S, Óunpuu S et al. Effect if potentially modifiable risk factors associated with myocardial infarction in 52 countries (the INTERHEART study): case-control-study. Lancet 2004; 364 (9438): 937-952

${ }^{8}$ WHO MONICA Project Principal Investigators. The World Health Organization MONICA Project: a major international collaboration. J Clin Epidemiol 1988; 34: 105 - 114

${ }^{9}$ Hense HW, Filipiak B, Döring A et al. Ten-year trends of cardiovascular risk factors in the MONICA Augsburg Region in southern Germany. Results from the 1984/85, 1989/90, and 1994/95 Surveys. CVD Prevention 1998; 1 : $318-327$

${ }^{10}$ Helmert U, Buitkamp M. Die Veränderung des Rauchverhaltens in Deutschland von 1985 bis 2002. Das Gesundheitswesen 2004; 66: $102-106$

${ }^{11}$ WHO MONICA Project prepared by Molarius A, Parson RW, Dobson AJ et al. Trends in cigarette smoking in 36 populations from the early 1980 s to the mid-1990 s: Findings from the WHO MONICA Project. Am J Public Health 2001; 91: 206-212

12 Stieber J, Härtel U, Heller WD et al. Smoking habits and attitude to smoking in the study population of the MONICA project Augsburg. Soz Präventivmed 1988; 33: $22-26$

${ }^{13}$ Härtel U, Stieber J, Keil U. Social relations and smoking behavior: Results from the first MONICA Survey Augsburg. Soz Präventivmed 1988; 33: $27-31$

${ }^{14}$ Härtel U, Stieber J, Keil U. Der Einfluß von Ausbildung und beruflicher Position auf Veränderungen im Zigarettenrauchen und Alkoholkonsum: Ergebnisse der MONICA Augsburg Kohortenstudie. Soz Präventivmed 1993; 38: 133-141

${ }^{15}$ Maziak W, Hense HW, Döring A et al. Ten-year trends in smoking behaviour among adults in southern Germany. Int J Tuberc Lung Dis 2002; 6 (9): 824-830

${ }^{16}$ Ladwig KH, Baumert J, Löwel $\mathrm{H}$ et al. Contemplating to quit current smoking status: Differences in behavioural and psychosocial patterns in a population-based cohort of current smokers. Prev Med (in press) 